\title{
Estimativa da erodibilidade pela desagregação por ultra-som e atributos de solos com horizonte $B$ textural
}

\author{
Marcos Aurélio Carolino de Sá(1), José Maria de Lima ${ }^{(2)}$, Nilton Curi(2), João Aguilar Massaroto(2) \\ e João José Granate de Sá e Mello Marques(2)
}

(1)Embrapa Cerrados, Caixa Postal 08223, CEP 73301-970 Planaltina, DF. E-mail: carolino@cpac.embrapa.br (2)Universidade Federal de Lavras, Dep. de Ciência do Solo, Caixa Postal 37, CEP 37200-000 Lavras, MG. E-mail: jmlima@ufla.br, niltcuri@ufla.br, jamassaroto@yahoo.com.br, jmarques@ufla.com.br

Resumo - A erodibilidade de solos é um fator importante na estimativa das perdas por erosão. Este fator é uma expressão da combinação de atributos do solo, os quais possibilitam sua estimativa por meio de equações. O objetivo deste trabalho foi medir atributos químicos e mineralógicos que, combinados com índices de estabilidade de agregados determinados por ultra-som, pudessem ser utilizados como variáveis em modelos na estimativa da erodibilidade de solos com horizonte B textural do Brasil. Estes atributos foram determinados em 22 solos de erodibilidade conhecida, medida diretamente em parcelas no campo. Atributos de 21 dos solos foram utilizados no ajuste dos modelos. Um dos solos (Argissolo Vermelho-Amarelo) foi escolhido ao acaso para teste. De 96 variáveis, 15 foram incluídas nos modelos de estimativa da erodibilidade. A maioria delas é representada por índices de desagregação por sonificação de amostras do horizonte A. Foram obtidos quatro modelos para estimar a erodibilidade, com $\mathrm{R}^{2}$ variando entre $0,83^{* *}$ e $0,91^{* *}$. A erodibilidade pode ser estimada com base na estabilidade de agregados por ultra-som.

Termos para indexação: USLE, estabilidade de agregados, química, mineralogia.

\section{Estimating soil erodibility from sonication indexes and other attributes of textural B horizon soils}

\begin{abstract}
Soil erodibility is an important factor for estimating soil erosion losses. This factor is an expression of combined soil attributes, which make possible its estimation by equations. The objective of this study was to measure some chemical and mineralogical attributes, and to combine them with aggregate stability indexes from sonication analysis, in equations in order to estimate soil erodibility of textural B horizon soils from Brazil. These attributes were measured for 22 soils that had their erodibility measured from field plots. Attributes of 21 soils were used to adjust the equations. One of the soils (Red-Yellow Argisol) was used to test the equations. From 96 variables, 15 were significantly correlated to soil erodibility. Most of them are represented by the disruption indexes from sonication analysis of A horizon samples. This study resulted in four equations to estimate soil erodibility, whose $\mathrm{R}^{2}$ ranged from $0.83^{* *}$ to $0.91^{* *}$. The erodibility can be estimated by aggregate stability indices based on sonication analysis.
\end{abstract}

Index terms: USLE, aggregate stability, chemistry, mineralogy.

\section{Introdução}

A erodibilidade (fator K) é uma das variáveis da Equação Universal de Perdas de Solo (USLE) que expressa, quantitativamente, a suscetibilidade do solo à erosão hídrica. Este fator depende de atributos como capacidade de infiltração de água e resistência à desagregação e ao transporte de partículas e pequenos agregados.

$\mathrm{O}$ fator $\mathrm{K}$ pode ser determinado diretamente no campo, sob chuva natural ou simulada, o que demanda tempo e altos custos, principalmente no primeiro caso. Também pode ser estimado por meio de modelos matemáticos que utilizam, como variáveis, atributos relacionados direta ou indiretamente com a resistência do solo à erosão (Denardin, 1990; Marques et al., 1997b; Silva et al., 1999).

A erodibilidade é um fator complexo e dependente da interação de múltiplos atributos (Silva et al., 1999) e por isso, torna-se difícil, estabelecer uma relação de causa e efeito entre as variáveis utilizadas nos modelos e a erodibilidade (Denardin, 1990; Marques et al., 1997b; Silva et al., 1999). Segundo Angulo (1983), a estabilidade de agregados possui boa correlação com a erodibilidade.

Nos últimos anos, pesquisas têm sido feitas visando à determinação da estabilidade de agregados mediante a 
aplicação de energia ultra-sônica (Fuller \& Goh, 1992; Raine \& So, 1993, 1994; Tippkötter, 1994; Sá et al., 1999, 2000b, 2002), cuja vantagem é a mensuração da quantidade de energia aplicada à amostra (Raine \& So, 1993).

Por meio dessa técnica, é possível conhecer a resistência que um solo oferece à desagregação, quando submetido a diferentes níveis de energia, até atingir a dispersão total. Índices de desagregação podem ser avaliados isoladamente ou em curvas de desagregação, cujo comportamento é um reflexo da estabilidade de agregados (Sá et al., 1999, 2002). Dessa forma, o conhecimento da energia associada à desagregação do solo torna a estabilidade de agregados determinada por ultrasom potencialmente promissora na determinação da erodibilidade.

O objetivo deste trabalho foi medir atributos químicos e mineralógicos que, combinados com índices de estabilidade de agregados por ultra-som, pudessem ser utilizados como variáveis em modelos para estimativa da erodibilidade de solos com horizonte B textural do Brasil.

\section{Material e Métodos}

Foram utilizadas amostras dos horizontes A e B de 23 solos de diversas regiões brasileiras cujos atributos estão mostrados na Tabela 1. A atualmente o solo 8 é enquadrado como tendo horizonte B plânico e o 20 como tendo horizonte B nítico (Embrapa, 1999), variações do abrangente horizonte $\mathrm{B}$ textural presente em todos os demais solos estudados. Os solos de 1 a 22 possuem a erodibilidade (fator K da USLE) determinada diretamente no campo (Tabela 2).

$\mathrm{Na}$ terra fina secada ao ar (TFSA), foi determinado o ponto de efeito salino nulo (PESN), conforme Zelasny et al. (1996), com modificações descritas em Sá (2002), em triplicata. O potencial de superfície foi calculado pela equação $\psi_{0}=59,1 \times\left(\mathrm{PESN}-\mathrm{pH}_{\text {água }}\right)$, conforme Raij (1973). O $\triangle \mathrm{pH}$ foi determinado segundo Embrapa (1997) e o PCZ (ponto de carga zero) foi estimado pela equação $\mathrm{PCZ}=\left(2 \times \mathrm{pH}_{\mathrm{KCl}}\right)-\mathrm{pH}_{\text {água }}$ (Uehara, 1988).

Os teores de $\mathrm{Fe}_{2} \mathrm{O}_{3}$ extraído pelo oxalato ácido de amônio e pelo ditionito-citrato-bicarbonato de sódio

Tabela 1. Classificação antiga, atual e localização dos solos estudados.

\begin{tabular}{|c|c|c|c|c|}
\hline \multicolumn{2}{|c|}{ Solo Classificação antiga } & \multirow{2}{*}{$\begin{array}{l}\text { Classificação atual } \\
\text { Argissolo Amarelo eutrófico abrúptico }\end{array}$} & \multirow{2}{*}{$\frac{\text { Localização }}{\text { Fortaleza, CE }}$} & \multirow{2}{*}{$\frac{\mathrm{K}\left[\mathrm{t} \mathrm{h}(\mathrm{MJ} \mathrm{mm})^{-1}\right]}{0,045^{(1)}}$} \\
\hline 1 & Podzólico Amarelo & & & \\
\hline 2 & Podzólico Vermelho-Escuro & Alissolo Crômico Argilúvico típico & Santa Maria, RS & $0,024^{(2)}$ \\
\hline 3 & Podzólico Vermelho-Escuro & Argissolo Vermelho distrófico típico & Eldorado do Sul, RS & $0,032^{(3)}$ \\
\hline 4 & Podzólico Vermelho-Amarelo & Argissolo Vermelho-Amarelo distrófico típico & Viçosa, MG & $0,027^{(4)}$ \\
\hline 5 & Podzólico Vermelho-Amarelo & Argissolo Vermelho-Amarelo eutrófico típico & Alagoa Grande, PB & $0,032^{(5)}$ \\
\hline 6 & Podzólico Vermelho-Escuro & Argissolo Vermelho eutrófico abrúptico plíntico & Itapororoca, $\mathrm{PB}$ & $0,018^{(5)}$ \\
\hline 7 & Bruno não Cálcico & Luvissolo Hipocrômico órtico planossólico & Gurinhém, PB & $0,032^{(5)}$ \\
\hline 8 & Solonetz Solodizado & Planossolo Nátrico órtico vértico & Boa Vista, PB & $0,012^{(6)}$ \\
\hline 9 & Bruno não Cálcico & Luvissolo Crômico órtico típico & Patos, PB & $0,008^{(7)}$ \\
\hline 10 & Podzólico Vermelho-Escuro & Luvissolo Crômico pálico típico & Patos, PB & $0,004^{(6)}$ \\
\hline 11 & Podzólico Vermelho-Amarelo & Argissolo Vermelho-Amarelo eutrófico típico & Tavares, PB & $0,025^{(7)}$ \\
\hline 12 & Podzólico Vermelho-Escuro & Argissolo Vermelho eutrófico típico & Teixeira, $\mathrm{PB}$ & $0,008^{(7)}$ \\
\hline 13 & Podzólico Vermelho-Amarelo & Alissolo Hipocrômico argilúvico típico & Alagoa Nova, PB & $0,031^{(5)}$ \\
\hline 14 & Bruno não Cálcico & Luvissolo Crômico órtico lítico & Casserengue, $\mathrm{PB}$ & $0,009^{(6)}$ \\
\hline 15 & Podzólico Vermelho-Amarelo & Argissolo Vermelho-Amarelo eutrófico típico & Glória do Goitá, PE & $0,014^{(8)}$ \\
\hline 16 & Podzólico Vermelho-Amarelo & Alissolo Crômico argilúvico típico & Morretes, PR & $0,004^{(9)}$ \\
\hline 17 & Podzólico Vermelho-Amarelo & Argissolo Vermelho-Amarelo eutrófico típico & Sete Lagoas, MG & $0,033^{(10)}$ \\
\hline 18 & Podzólico Vermelho-Escuro & Alissolo Crômico argilúvico típico & Santa Maria, RS & $0,034^{(3)}$ \\
\hline 19 & Podzólico Vermelho-Escuro & Argissolo Vermelho eutrófico arênico & Pindorama, SP & $0,004^{(11)}$ \\
\hline 20 & Podzólico Vermelho-Amarelo & Nitossolo Háplico eutrófico típico & Mococa, SP & $0,023^{(12)}$ \\
\hline 21 & Podzólico Vermelho-Escuro & Argissolo Vermelho eutrófico típico & Itaguaí, RJ & $0,028^{(13)}$ \\
\hline 22 & Podzólico Vermelho-Amarelo & Argissolo Vermelho-Amarelo eutrófico abrúptico plíntico & Quixadá, CE & $0,008^{(1)}$ \\
\hline 23 & Podzólico Vermelho-Escuro & Argissolo Vermelho eutrófico típico & Patu, RN & - \\
\hline
\end{tabular}

${ }^{(1)}$ Silva (1994). ${ }^{(2)}$ Dal Conte (1982). ${ }^{(3)}$ Levien, citado por Denardin (1990). ${ }^{(4)}$ Resck et al. (1981). ${ }^{(5)}$ Rodrigues do Ó (1986). ${ }^{(6)}$ Silva \& Andrade (1994). ${ }^{(7)}$ Silva et al. (1986). ${ }^{(8)}$ Campos Filho et al. (1992). ${ }^{(9)}$ Embrapa, citado por Marques (1996). ${ }^{(10)}$ Marques (1996). ${ }^{(11)}$ Carvalho et al. (1994), citado por Marques (1996). ${ }^{(12)}$ Carvalho et al. (1989). ${ }^{(13)}$ Leprun, citado por Denardin (1990). 
(DCB) foram determinados na fração argila, conforme Embrapa (1997) e corrigidos para TFSA. Caulinita e gibbsita foram quantificadas na fração argila por análise térmica diferencial (ATD), e a atividade da fração argila (Targ) foi calculada conforme Embrapa (1999), sem a correção para C orgânico.

A estabilidade de agregados foi determinada por sonificação, nas classes de agregados entre 4,76 e 7,93 mm e menores que $2 \mathrm{~mm}$ (TFSA), com um aparelho Misonix, modelo XL2020 ${ }^{\mathrm{TM}}$, operando a $20 \mathrm{kHz}$ e fornecendo uma potência nominal de $154 \mathrm{~W}$, que correspondia a uma potência real de $120,91 \pm 3,27 \mathrm{~W}$, calculada conforme Sá et al. (2000a, 2001). As sonificações foram feitas em béquer com $200 \mathrm{~mL}$ de água destilada, na relação solo:água de 1:40. Os níveis de energia específica aplicados às amostras foram calculados pela equação $\mathrm{EA}=\left[\mathrm{P}_{\mathrm{c}} \mathrm{t}_{\mathrm{s}}\right] / \mathrm{v}$, em que, EA é a energia ultra-sônica aplicada à amostra $\left(\mathrm{J} \mathrm{mL}^{-1}\right) ; \mathrm{P}_{\mathrm{c}}$ é a potência (W) emitida pelo aparelho e calculada mediante calibração; $\mathrm{t}_{\mathrm{s}}$ é o tempo de sonificação (s); v é o volume da suspensão (mL), conforme Sá et al. (2000a, 2001).
A ponta da haste do aparelho, com comprimento de $175 \mathrm{~mm}$ e diâmetro de $19 \mathrm{~mm}$, foi inserida na água a uma profundidade de $47 \mathrm{~mm}$. Conforme testes preliminares, a temperatura da suspensão aumenta linearmente com o tempo de sonificação, sendo necessário colocar o béquer em banho de gelo para tempos acima de 180 segundos, mantendo-se a temperatura da suspensão abaixo de $35^{\circ} \mathrm{C}$, durante a sonificação, de acordo com Raine \& So (1994). Após cada sonificação, as amostras foram passadas em peneira de malha de $0,053 \mathrm{~mm}$. O material retido nesta peneira (MRP) foi secado em estufa por 24 horas a $105^{\circ} \mathrm{C}$ e pesado. Os teores de silte + argila (sólidos que passaram pela peneira) foram obtidos por diferença: silte + argila $=$ amostra original (5 g) - MRP. Este procedimento possibilitou, em cada nível de energia específica aplicado, o cálculo do índice de desagregação (ID), obtido pela relação (silte + argila dispersa em g)/amostra original (g), e também o índice de desagregação normalizado (IDN) pela relação ID/ID máximo (Sá et al., 1999). O primeiro representa o efeito da estabilidade de agregados e granulometria, e o segundo, apenas da estabilidade de

Tabela 2. Atributos dos horizontes A e B dos solos estudados.

\begin{tabular}{|c|c|c|c|c|c|c|c|c|c|c|c|}
\hline \multirow[t]{2}{*}{ Solo } & \multicolumn{4}{|c|}{ Horizonte A } & \multicolumn{7}{|c|}{ Horizonte B } \\
\hline & Areia & Silte & Argila & Corg. ${ }^{(1)}$ & Areia & Silte & Argila & Corg. ${ }^{(1)}$ & $\mathrm{V}(\%)^{(2)}$ & $\mathrm{Ki}^{(3)}$ & $\mathrm{Kr}^{(4)}$ \\
\hline 1 & 812 & 46 & 142 & 4 & 577 & 55 & 368 & 4 & 58 & 2,14 & 1,14 \\
\hline 2 & 479 & 303 & 218 & 13 & 238 & 235 & 527 & 6 & 25 & 2,25 & 1,03 \\
\hline 3 & 706 & 150 & 144 & 15 & 302 & 138 & 560 & 4 & 20 & 2,16 & 1,05 \\
\hline 4 & 481 & 141 & 378 & 14 & 238 & 125 & 637 & 6 & 49 & 1,82 & 0,84 \\
\hline 5 & 678 & 160 & 162 & 14 & 594 & 118 & 288 & 5 & 65 & 1,95 & 0,86 \\
\hline 6 & 666 & 144 & 190 & 7 & 437 & 166 & 397 & 3 & 52 & 2,13 & 0,97 \\
\hline 7 & 659 & 196 & 145 & 13 & 517 & 158 & 325 & 4 & 85 & 2,72 & 1,18 \\
\hline 8 & 769 & 120 & 111 & 4 & 471 & 62 & 467 & 6 & 81 & 2,62 & 1,15 \\
\hline 9 & 687 & 153 & 160 & 9 & 557 & 154 & 289 & 4 & 89 & 2,50 & 1,07 \\
\hline 10 & 615 & 194 & 191 & 9 & 555 & 195 & 250 & 4 & 76 & 1,89 & 0,85 \\
\hline 11 & 652 & 142 & 206 & 9 & 472 & 160 & 368 & 5 & 77 & 2,04 & 1,02 \\
\hline 12 & 563 & 129 & 308 & 11 & 397 & 107 & 496 & 7 & 51 & 2,01 & 0,95 \\
\hline 13 & 628 & 123 & 249 & 17 & 596 & 92 & 312 & 13 & 7 & 1,78 & 0,93 \\
\hline 14 & 665 & 202 & 133 & 7 & 431 & 128 & 441 & 4 & 80 & 2,61 & 1,11 \\
\hline 15 & 657 & 117 & 226 & 2 & 540 & 150 & 310 & 3 & 66 & 2,10 & 1,03 \\
\hline 16 & 257 & 368 & 375 & 28 & 179 & 164 & 657 & 3 & 2 & 2,03 & 0,91 \\
\hline 17 & 110 & 366 & 524 & 21 & 54 & 237 & 709 & 9 & 52 & 2,00 & 1,01 \\
\hline 18 & 682 & 106 & 212 & 9 & 436 & 111 & 453 & 7 & 14 & 2,07 & 1,00 \\
\hline 19 & 816 & 75 & 109 & 6 & 601 & 58 & 341 & 4 & 74 & 2,02 & 0,97 \\
\hline 20 & 532 & 150 & 318 & 13 & 368 & 188 & 444 & 11 & 74 & 1,78 & 0,81 \\
\hline 21 & 622 & 110 & 268 & 12 & 405 & 89 & 506 & 3 & 55 & 2,09 & 1,60 \\
\hline 22 & 829 & 60 & 111 & 5 & 565 & 94 & 341 & 4 & 75 & 2,35 & 1,18 \\
\hline 23 & 489 & 358 & 153 & 12 & 276 & 456 & 268 & 2 & 83 & 2,07 & 0,98 \\
\hline
\end{tabular}

${ }^{(1)}$ Carbono orgânico. ${ }^{(2)}$ Saturação por bases. ${ }^{(3)}$ Relação molecular $\mathrm{SiO}_{2} / \mathrm{Al}_{2} \mathrm{O}_{3}$. ${ }^{(4)}$ Relação molecular $\mathrm{SiO}_{2} / \mathrm{Al}_{2} \mathrm{O}_{3}+\mathrm{Fe}_{2} \mathrm{O}_{3}$. 
agregados, conforme Sá (2002). O tempo zero de sonificação, consistiu em colocar a amostra no béquer, completar o volume até $200 \mathrm{~mL}$ com água destilada e passar imediatamente pela peneira, a fim de quantificar a desagregação causada pelo manuseio da amostra.

Em virtude da quantidade limitada de agregados da classe 7,93 a 4,76 mm, foi possível fazer apenas uma replicata em relação às amostras de horizonte $\mathrm{A}$ dos solos 8,10 e 22 e duas replicatas do horizonte A do solo 1 e horizonte $\mathrm{B}$ dos solos 10 e 23 . Foram feitas três replicatas para as demais amostras. Em cada replicata, cujo peso foi equivalente a $5 \mathrm{~g}$ de agregados secados em estufa a $105^{\circ} \mathrm{C}$, foi feito um pré-umedecimento por capilaridade (Sá et al., 1999). Elas foram, então, submetidas aos tempos de sonificação de $0,5,15,30,60$, $300,900,1.800$ e $2.700 \mathrm{~s}$, o que corresponde, respectivamente, às energias específicas aplicadas de $0,0,3,0$, $9,1,18,1,36,3,181,4,544,1,1.088,2$ e $1.682,3 \mathrm{~J} \mathrm{~mL}^{-1}$. Uma vez que alguns solos estudados apresentam considerável teor de cascalho, no cálculo dos índices de desagregação foi descontada a contribuição desta fração. Isso foi feito separando-se do MRP, após a sonificação, as partículas maiores que $2 \mathrm{~mm}$ e descontando-se o seu peso do peso total da amostra inicial.

Foram então construídas curvas de desagregação, plotando-se no eixo das abscissas (X) a energia aplica$\mathrm{da}$, em $\mathrm{J} \mathrm{mL}^{-1}$, e no eixo das ordenadas (Y), os índices de desagregação, normalizados ou não, ajustando-se modelos do tipo $\mathrm{Y}=\mathrm{X} /(\mathrm{a}+\mathrm{bX})$, sendo a e $\mathrm{b}$ coeficientes específicos para cada solo. Foi calculado um índice, que expressa a relação entre os coeficientes b/a, e a estabilidade de agregados, com base no comportamento das curvas de desagregação, conforme demonstrado por Sá et al. (1999).

$\mathrm{Na}$ classe de agregados menores que $2 \mathrm{~mm}$, todas as análises foram realizadas em triplicatas. Em cada amostra, foram utilizados o equivalente a $5 \mathrm{~g}$ de agregados secados em estufa a $105^{\circ} \mathrm{C}$. Foi feito um préumedecimento conforme Fuller \& Goh (1992), que consistiu em colocar os agregados secados em béquer de $250 \mathrm{~mL}$, o qual foi colocado em um suporte, em posição inclinada $\left(45^{\circ}\right)$ abaixo de uma bureta, sendo gotejada água destilada, lentamente (aproximadamente $2 \mathrm{~mL}$ por minuto), sobre a parede do béquer, promovendo umedecimento gradativo dos agregados. Quando estes já estavam cobertos por água destilada, o volume do béquer foi completado até $200 \mathrm{~mL}$. As amostras foram então submetidas aos seguintes tempos de sonificação:
$0,5,15,30,60,120,180,240$ e 300 s. Cada tempo de sonificação corresponde, respectivamente, às energias específicas aplicadas de $0,0,3,0,9,1,18,1,36,3,72,5$, $108,8,145,1$ e $181,4 \mathrm{~J} \mathrm{~mL}^{-1}$.

Testes preliminares mostraram que os índices de desagregação se estabilizavam a partir de $181,5 \mathrm{~J} \mathrm{~mL}^{-1}$, dispensando, portanto, o uso de níveis mais elevados de energia. Os demais procedimentos de sonificação e cálculo dos índices ID e IDN e o ajuste das curvas de desagregação seguiram os mesmos passos utilizados em relação aos agregados da classe 7,93 a 4,76 mm.

No ajuste dos modelos, foram utilizadas variáveis baseadas em atributos dos solos (PESN, $\psi_{0}$, PCZ estimado, $\triangle \mathrm{pH}$, Targ, $\mathrm{Fe}_{2} \mathrm{O}_{3}$-DCB, $\mathrm{Fe}_{2} \mathrm{O}_{3}$-oxalato e $\mathrm{Ct}$, nos horizontes A e B) e índices de desagregação por ultrasom (ID e IDN, nos horizontes A e B e nas duas classes de agregados e b/a, para ambos os casos), num total de 96 variáveis, as quais foram utilizadas no ajuste de modelos para predição da erodibilidade, por regressão linear múltipla passo a passo (backward-stepwise), adotando-se 5\% como nível de significância máximo para inclusão de uma variável no modelo. A aceitação dos modelos foi feita mediante interpretação de $\mathrm{R}^{2}, \mathrm{R}^{2}$ ajustado e erro-padrão da estimativa (EPE). O solo 5, cuja erodibilidade determinada em campo também é conhecida, não foi incluído no ajuste dos modelos, sendo escolhido ao acaso para teste. Assim, sua erodibilidade estimada foi comparada com a erodibilidade conhecida. Também foi estimada a erodibilidade do solo 23 e comparada com a erodibilidade estimada por Marques (1996).

\section{Resultados e Discussão}

Conforme constatado por Denardin (1990), Marques et al. (1997b) e Silva et al. (1999), nenhuma das variáveis, isoladamente, foi capaz de predizer razoavelmente a erodibilidade. Assim, das 96 variáveis obtidas, 15 apresentaram nível de significância adequado e foram, portanto, incluídas nos modelos. As variáveis excluídas não serão discutidas neste trabalho, mas podem ser consultadas em Sá (2002). Os respectivos valores mínimo, médio e máximo observados em cada variável no conjunto de solos estudados, em que o fator K determinado diretamente no campo varia entre $0,004 \mathrm{e}$ $0,045 \mathrm{th}(\mathrm{MJ} \mathrm{mm})^{-1}$, podem ser observados na Tabela 3. Desta maneira, em estimativas futuras do fator $\mathrm{K}$ utilizando os modelos propostos, valores obtidos fora desta faixa devem ser interpretados com cautela. A mesma 
observação é válida para solos que apresentem valores de $\mathrm{X}_{1}$ a $\mathrm{X}_{15}$ fora do intervalo observado.

O número de variáveis oriundas do horizonte $\mathrm{A}$ (12) é superior ao do B (3). As variáveis $\mathrm{X}_{1}$ a $\mathrm{X}_{10}$ são índices de estabilidade de agregados por ultra-som e todas são da classe $<2 \mathrm{~mm}$ do horizonte $\mathrm{A}$. $\mathrm{O}$ fato de apenas agregados desta classe apresentarem nível de significância adequado para fazer parte dos modelos se deve, provavelmente, à proximidade de tamanho com a classe 0,06-2,00 mm, que, conforme Tippkötter (1994), é a que melhor expressa o comportamento do solo perante a erosão hídrica. Também são do horizonte $\mathrm{A}$ as variáveis $\mathrm{X}_{11}$ e $\mathrm{X}_{14}$, respectivamente, Targ e $\mathrm{Ct}$. As variáveis $\mathrm{X}_{12}$, $\mathrm{X}_{13}$ e $\mathrm{X}_{15}$ são oriundas do horizonte $\mathrm{B}$, respectivamente, Targ, $\mathrm{Fe}_{2} \mathrm{O}_{3}$-DCB e Ct.

A presença de variáveis do horizonte $\mathrm{B}$ nos modelos mostra a importância do horizonte subsuperficial no processo erosivo deste grupamento de solos, semelhante ao observado por Marques et al. (1997b). Isso pode ser uma das causas da inadequação de modelos propostos no passado, que utilizavam apenas variáveis do horizonte A na estimativa do fator K deste grupamento de solos, conforme demonstrado por Marques et al. (1997a).

$\mathrm{O}$ fato de a maioria das variáveis selecionadas ser obtida em níveis de energia ultra-sônica mais baixos confirma as observações de Raine \& So (1993) de que altos níveis de energia, nos quais ocorre a desagrega- ção total do solo, fornecem poucas informações sobre o comportamento do solo no campo, no que diz respeito às relações entre estabilidade de agregados e resistência à erosão. Entretanto, fica difícil estabelecer as relações de causa e efeito entre cada uma delas e os valores de erodibilidade, o que também foi observado por Denardin (1990) e Silva et al. (1999). Segundo esses autores, a erodibilidade depende tanto da capacidade de infiltração de água do solo quanto da sua capacidade de resistir à desagregação e também ao transporte causado pela energia das gotas de chuva e do escorrimento superficial.

As variáveis selecionadas têm participação, pelo menos indireta, no processo erosivo, pois, conforme Fuller \& Goh (1992), a argila dispersa pelo ultra-som é um atributo adequado na avaliação da quebra de agregados. Além disso, segundo Gregorich et al. (1988), essa argila, quando dispersa em baixos níveis de energia, provém de material que liga microagregados entre si, formando macroagregados. Assim, as frações silte + argila dos índices de desagregação (variáveis $\mathrm{X}_{1}$ a $\mathrm{X}_{9}$ ), quando obtidas em níveis de energia mais baixos, seriam oriundas da quebra de macroagregados, pois, quanto maior o tamanho dos agregados, mais fracas as forças que unem as partículas (Oades, 1993). Pode-se então inferir que, durante chuva erosiva, este material pode estar sendo salpicado, se alojando entre unidades maiores, causan-

Tabela 3. Descrição das variáveis incluídas nos modelos e respectivos valores mínimo, médio e máximo ${ }^{(1)}$.

\begin{tabular}{|c|c|c|c|c|}
\hline \multirow[t]{2}{*}{ Variável } & \multirow[t]{2}{*}{ Descrição } & \multicolumn{3}{|c|}{ Valor } \\
\hline & & Mínimo & Médio & Máximo \\
\hline $\mathrm{K}$ & Erodibilidade - fator $\mathrm{K}$ da USLE [t h $\left(\mathrm{MJ} \mathrm{mm}^{-1}\right)$ ] & 0,004 & 0,020 & 0,045 \\
\hline$X_{1}$ & ID obtido a $3,0 \mathrm{~J} \mathrm{~mL}^{-1}$ & 0,039 & 0,113 & 0,226 \\
\hline$X_{2}^{1}$ & ID obtido a $9,1 \mathrm{~J} \mathrm{~mL}^{-1}$ & 0,049 & 0,137 & 0,265 \\
\hline $\mathrm{X}_{3}^{2}$ & ID obtido a $18,1 \mathrm{~J} \mathrm{~mL}^{-1}$ & 0,084 & 0,170 & 0,347 \\
\hline $\mathrm{X}_{4}^{3}$ & ID obtido a $36,3 \mathrm{~J} \mathrm{~mL}^{-1}$ & 0,083 & 0,214 & 0,496 \\
\hline$X_{5}^{4}$ & ID obtido a $145,1 \mathrm{~J} \mathrm{~mL}^{-1}$ & 0,149 & 0,380 & 0,907 \\
\hline$X_{6}^{5}$ & IDN obtido a $3,0 \mathrm{~J} \mathrm{~mL}^{-1}$ & 0,138 & 0,315 & 0,595 \\
\hline $\mathrm{X}_{7}^{6}$ & IDN obtido a $9,1 \mathrm{~J} \mathrm{~mL}^{-1}$, & 0,169 & 0,386 & 0,724 \\
\hline $\mathrm{X}_{8}$ & IDN obtido a $18,1 \mathrm{~J} \mathrm{~mL}^{-1}$ & 0,249 & 0,474 & 0,772 \\
\hline$X_{9}^{8}$ & IDN obtido a $108,8 \mathrm{~J} \mathrm{~mL}^{-1}$ & 0,864 & 0,953 & 0,997 \\
\hline $\mathrm{X}_{10}^{9}$ & Índice b/a (curva entre 0 e $181,4 \mathrm{~J} \mathrm{~mL}^{-1}$ ) & 0,034 & 0,120 & 0,394 \\
\hline$X_{11}^{10}$ & Atividade da fração argila no horizonte A & 13,230 & 40,710 & 89,970 \\
\hline$X_{12}^{11}$ & Atividade da fração argila no horizonte B & 6,440 & 25,990 & 62,280 \\
\hline$X_{13}^{12}$ & $\mathrm{Fe}_{2} \mathrm{O}_{3}$ extraído pelo DCB no horizonte $\mathrm{B}\left(\mathrm{g} \mathrm{kg}^{-1}\right)$ & 5,610 & 25,920 & 69,130 \\
\hline$X_{14}^{13}$ & Caulinita no horizonte $\mathrm{A}\left(\mathrm{g} \mathrm{kg}^{-1}\right)$ & 18,310 & 87,050 & 221,930 \\
\hline$X_{15}^{14}$ & Caulinita no horizonte $\mathrm{B}\left(\mathrm{g} \mathrm{kg}^{-1}\right)$ & 56,200 & 185,250 & 429,530 \\
\hline
\end{tabular}

${ }^{(1)}$ ID: índice de desagregação; IDN: índice de desagregação normalizado; as variáveis de $\mathrm{X}_{1}$ a $\mathrm{X}_{10}$ são obtidas de agregados da classe <2 mm do horizonte A. 
do um selamento superficial, o que diminui a permeabilidade e aumenta a erosão (Roth \& Eggert, 1994). A variável $X_{10}$ (índice b/a) representa a estabilidade de agregados como um todo, com base no comportamento da curva de desagregação (Sá et al., 1999).

Conforme Sá et al. (2002), índices de desagregação obtidos a 36,3 e 72,5 $\mathrm{J} \mathrm{mL}^{-1}$ apresentam melhor sensibilidade em detectar as diferenças na estabilidade de agregados promovidas pelo uso do solo. Entretanto, neste trabalho, índices obtidos a $72,5 \mathrm{~J} \mathrm{~mL}^{-1}$ não apresentaram nível de significância adequado a fim de serem incluídos nos modelos. Provavelmente, isso se deve ao fato de os autores terem avaliado um Latossolo sob diferentes usos, enquanto neste trabalho, são estudados apenas solos com horizonte B textural. Outras variáveis $\left(\mathrm{X}_{11}\right.$ a $\left.\mathrm{X}_{15}\right)$, que são atributos como atividade da fração argila, $\mathrm{Fe}_{2} \mathrm{O}_{3}$-DCB e Ct, em ambos os horizontes, estão associadas à estabilidade de agregados e tipo de estrutura, o que, de certa forma, influencia a permeabilidade (Ferreira et al., 1999).

Foram escolhidos os modelos de utilização mais prática, os quais apresentaram altos valores de $\mathrm{R}^{2}$, significativos a menos de 1\%, em geral (Tabela 4), de mesma magnitude dos valores encontrados por Denardin (1990),

Tabela 4. Variáveis e coeficientes dos modelos ajustados por regressão passo a passo (backward-stepwise) para predição da erodibilidade (fator $\mathrm{K})^{(1)}$.

\begin{tabular}{lcccc}
\hline Variável & Modelo 1 & Modelo 2 & Modelo 3 & Modelo 4 \\
\hline Constante & $-0,4807$ & $-0,4138$ & $-0,1944$ & $-0,1910$ \\
$\mathrm{X}_{1}$ & 3,8387 & 3,6302 & - & - \\
$\mathrm{X}_{2}$ & $-4,3743$ & $-4,2297$ & - & - \\
$\mathrm{X}_{3}$ & 0,7721 & 0,9215 & - & - \\
$\mathrm{X}_{4}$ & $-0,2797$ & $-0,2614$ & $-0,2672$ & $-0,2649$ \\
$\mathrm{X}_{5}$ & 0,2434 & 0,1696 & 0,2848 & 0,2741 \\
$\mathrm{X}_{6}$ & $-1,3008$ & $-1,2295$ & - & - \\
$\mathrm{X}_{7}$ & 1,6139 & 1,5583 & - & - \\
$\mathrm{X}_{8}$ & $-0,2715$ & $-0,3187$ & - & - \\
$\mathrm{X}_{9}$ & 0,4574 & 0,4202 & 0,1839 & 0,1974 \\
$\mathrm{X}_{10}$ & $-0,2058$ & $-0,1937$ & - & - \\
$\mathrm{X}_{11}$ & 0,0002 & - & - & - \\
$\mathrm{X}_{12}$ & - & - & 0,0003 & - \\
$\mathrm{X}_{13}$ & - & - & $-0,0008$ & $-0,0007$ \\
$\mathrm{X}_{14}$ & - & - & $-0,0007$ & $-0,0006$ \\
$\mathrm{X}_{15}$ & - & - & 0,0003 & 0,0003 \\
\hline $\mathrm{R}^{2}$ & $0,91^{* *}$ & $0,85^{* *}$ & $0,87 * *$ & $0,83^{* *}$ \\
$\mathrm{R}^{2}$ ajustado & $0,81^{* *}$ & $0,72^{* *}$ & $0,80 * *$ & $0,76^{* *}$ \\
$\mathrm{EPE}$ & 0,0053 & 0,0065 & 0,0054 & 0,0059 \\
$\mathrm{n}$ & 21 & 21 & 21 & 21 \\
\hline
\end{tabular}

${ }^{(1)} \mathrm{X}_{1}$ a $\mathrm{X}_{15}$ : variáveis; $\mathrm{R}^{2}$ : coeficiente de determinação; EPE: erro padrão da estimativa [t $\left.\mathrm{h}(\mathrm{MJ} \mathrm{mm})^{-1}\right]$; $\mathrm{n}$ : no de solos utilizados no ajuste do modelo. **Significativo a $1 \%$ de probabilidade.
Marques et al. (1997b) e Silva et al. (1999). A maioria das variáveis incluídas nos modelos apresentou nível de significância menor que $1 \%$, exceto as variáveis $\mathrm{X}_{3} \mathrm{e}$ $\mathrm{X}_{11}$ no modelo $1, \mathrm{X}_{3} \mathrm{X}_{4}$ e $\mathrm{X}_{5}$ no modelo 2 e $\mathrm{X}_{12}$ no modelo 3 , que apresentaram níveis de significância menores do que $5 \%$.

Os modelos 1 e 2 utilizam apenas variáveis oriundas do horizonte A. No modelo 1, com exceção da variável $\mathrm{X}_{11}$ (atividade da fração argila), todas são índices de estabilidade de agregados por ultra-som. No modelo 2 , a retirada da variável $\mathrm{X}_{11}$ fez com que o $\mathrm{R}^{2}$ diminuísse de $0,91^{* *}$ para $0,85^{* *}$, sendo, neste caso, a erodibilidade estimada apenas a partir da estabilidade de agregados por ultra-som. Já os modelos 3 e 4 possuem menos variáveis, porém, algumas não são determinadas rotineiramente, como as variáveis $\mathrm{X}_{14}$ e $\mathrm{X}_{15}$, respectivamente, $\mathrm{Ct}$ nos horizontes $\mathrm{A}$ e $\mathrm{B}$, obtidas por análise térmica diferencial (ATD).

Os valores do fator $\mathrm{K}$ estimados pelos modelos estão próximos dos valores determinados no campo, em que os valores estimados estão distribuídos junto da linha 1:1 (Figura 1a). A precisão dos modelos pode ser melhor observada na Figura 1b. Em todos os casos, os resíduos padronizados ficaram entre 2 e -2 , o que é considerado um bom resultado, conforme Neter \& Wasserman (1974), sendo que, na maioria dos solos, os valores ficaram entre 1 e -1 .

No teste dos modelos, foi estimada a erodibilidade do solo 5, cujo valor determinado no campo é 0,032 t h $(\mathrm{MJ} \mathrm{mm})^{-1}$ (Tabela 5). Com relação a este solo, os valores estimados variaram entre 0,022 e $0,041 \mathrm{t} \mathrm{h}(\mathrm{MJ} \mathrm{mm})^{-1}$, estando a diferença entre o valor real e estimado, para todos os modelos, dentro da faixa de \pm 2 EPE para cada modelo, o que é considerado um bom resultado (Neter \& Wasserman, 1974). No solo 23, os valores estimados variaram entre 0,028 e $0,043 \mathrm{th}(\mathrm{MJ} \mathrm{mm})^{-1}$, abaixo do valor estimado por Marques (1996), que foi de 0,047 t h (MJ mm) ${ }^{-1}$. Porém, a erodibilidade deste solo, determinada de maneira direta, ainda não é conhecida.

Embora ainda não seja comum a determinação da estabilidade de agregados por ultra-som, a determinação dos índices de desagregação utilizados na predição do fator K não é trabalhosa, pois as sonificações variam de 30 a 300 segundos, considerando-se as especificações do aparelho utilizado. Do mesmo modo, o cálculo dos índices é também bastante prático, sendo, porém, necessária uma rigorosa padronização da ener- 

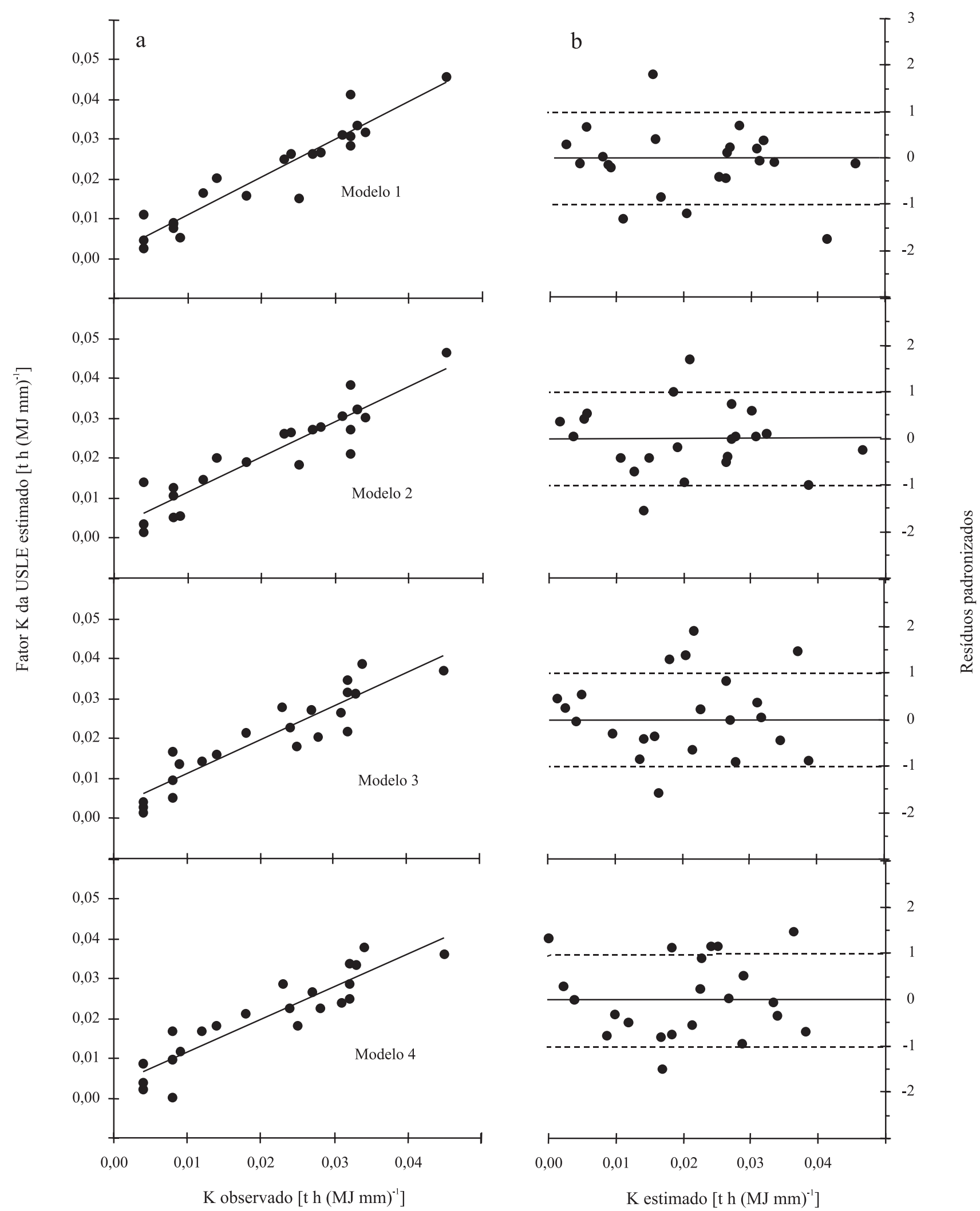

Figura 1. Relação entre os valores do fator K observados e estimados pelos modelos; o traço contínuo representa a linha 1:1 (a); resíduos padronizados, em que o traço pontilhado representa o erro-padrão da estimativa (b). 
Tabela 5. Comparação entre a erodibilidade (fator K) prevista pelos modelos propostos e a erodibilidade já conhecida do solo 5 e estimativa da erodibilidade do solo 23.

\begin{tabular}{|c|c|c|c|c|c|}
\hline \multirow[t]{2}{*}{ Solo } & \multirow[t]{2}{*}{ K conhecido } & \multicolumn{4}{|c|}{ K estimado pelos modelos } \\
\hline & & 1 & 2 & 3 & 4 \\
\hline & ---- & $-[\mathrm{t} \mathrm{h}(\mathrm{N}$ & $\left.\mathrm{mm})^{-1}\right]$ & - & ------ \\
\hline 5 & 0,032 & 0,041 & 0,038 & 0,022 & 0,025 \\
\hline 23 & $0,004^{(1)}$ & 0,047 & 0,043 & 0,028 & 0,030 \\
\hline
\end{tabular}

${ }^{(1)}$ Estimado indiretamente por Marques (1996).

gia aplicada. Na validação dos modelos, é importante a realização de trabalhos futuros visando estimar o fator $\mathrm{K}$ para solos com horizonte $\mathrm{B}$ textural que possuam a erodibilidade determinada de maneira direta, seja com chuva natural ou simulada, mas que não tomaram parte no presente trabalho. Importante também é a estimativa do fator $\mathrm{K}$ dos solos com horizonte $\mathrm{B}$ textural de diversas regiões do Brasil com atributos contrastantes, ou seja, teores extremos de argila, areia, silte, óxidos de ferro, alumínio, C orgânico, por exemplo, o que fornecerá informações sobre a real adequação dos modelos propostos.

\section{Conclusão}

A erodibilidade do solo pode ser estimada com base na estabilidade de agregados por ultra-som.

\section{Agradecimento}

Ao CNPq, pelo financiamento da pesquisa e pela concessão de bolsa aos autores Marcos Aurélio Carolino de Sá, José Maria de Lima, Nilton Curi, João Aguilar Massaroto e João José Granate de Sá e Mello Marques.

\section{Referências}

ANGULO, J.R. Relações entre a erodibilidade e algumas propriedades de solos brasileiros. 1983. 154p. Dissertação (Mestrado) - Universidade Federal do Paraná, Curitiba.

CAMPOS FILHO, O.R.; SILVA, I.F.; ANDRADE, A.P.; LEPRUN, J.C. Erosividade da chuva e erodibilidade do solo no agreste de Pernambuco. Pesquisa Agropecuária Brasileira, v.27, p.13631370, 1992.

CARVALHO, M.P.; LOMBARDI NETO, F.; VASQUES FILHO, J.; CATANEO, A. Índices de erosividade da chuva correlacionados com perdas de solo de um Podzólico Vermelho-Amarelo eutrófico textura argilosa/muito argilosa de Mococa (SP): Primeira aproximação do fator erodibilidade do solo. Revista Brasileira de Ciência do Solo, v.13, p.237-242, 1989.
DAL CONTE, F.M. Índice de erodibilidade de um solo Podzólico Vermelho-Amarelo, determinado sob chuva simulada. 1982. 67p. Dissertação (Mestrado) - Universidade Federal de Santa Maria, Santa Maria.

DENARDIN, J.E. Erodibilidade de solo estimada por meio de parâmetros físicos e químicos. 1990. 81p. Tese (Doutorado) Escola Superior de Agricultura Luiz de Queiroz, Piracicaba.

EMBRAPA. Centro Nacional de Pesquisa de Solos (Rio de Janeiro, RJ). Manual de métodos de análises de solo. 2.ed. Rio de Janeiro, 1997. 212p.

EMBRAPA. Centro Nacional de Pesquisa de Solos (Rio de Janeiro, RJ). Sistema brasileiro de classificação de solos. Brasília: Embrapa-SPI; Embrapa-CNPS, 1999. 412p.

FERREIRA, M.M.; FERNANDES, B.; CURI, N. Influência da mineralogia da fração argila nas propriedades físicas de latossolos da região sudeste do Brasil. Revista Brasileira de Ciência do Solo, v.23, p.515-524, 1999.

FULLER, L.G.; GOH, T.G. Stability-energy relationships and their application to aggregation studies. Canadian Journal of Soil Science, v.72, p.453-466, 1992.

GREGORICH, E.G.; KACHANOSKI, R.G.; VORONEY, R.P. Ultrasonic dispersion of aggregates: distribution of organic matter in size fractions. Canadian Journal of Soil Science, v.68, p.395403, 1988.

MARQUES, J.J.G. de S. e M. Estimativas e comparações dos fatores erosividade das chuvas e erodibilidade de solos com horizonte B textural no Brasil. 1996, 119p. Dissertação (Mestrado) - Universidade Federal de Lavras, Lavras.

MARQUES, J.J.G. de S. e M.; CURI, N.; FERREIRA, M.M.; LIMA, J.M. de; SILVA, M.L.N.; SÁ, M.A.C. de. Adequação de métodos indiretos para estimativa da erodibilidade de solos com horizonte B textural no Brasil. Revista Brasileira de Ciência do Solo, v.21, p.447-456, 1997a.

MARQUES, J.J.G. de S. e M.; CURI, N.; LIMA, J. M. de; FERREIRA, M.M.; SILVA, M.L.N.; FERREIRA, D.F. Estimativa da erodibilidade a partir de atributos de solos com horizonte B textural no Brasil. Revista Brasileira de Ciência do Solo, v.21, p.457465, $1997 \mathrm{~b}$.

NETER, J.; WASSERMAN, W. Applied linear statistical models: regression analysis of variance and experimental designs. Homewood: Richard D. Irwin Inc., 1974. 842p.

OADES, J.M. The role of biology in the formation, stabilization and degradation of soil structure. Geoderma, v.56, p.337-400, 1993.

RAIJ, B. van. Determinação do ponto de carga zero em solos. Bragantia, v.32, p.337-347, 1973 .

RAINE, S.R.; SO, B. An energy based parameter for the assessment of aggregate bond energy. Journal of Soil Science, v.44, p.249259, 1993.

RAINE, S.R.; SO, B. Ultrasonic dispersion of soil in water: the effect of suspension properties on energy dissipation and soil dispersion. Australian Journal of Soil Research, v.32, p.1157-1174, 1994.

RESCK, D.V.S.; FIGUEIREDO, M.S.; FERNANDES, B.; RESENDE, M.; SILVA, T.C.A. Erodibilidade de um Podzólico 
Vermelho-Amarelo câmbico distrófico fase terraço, localizado na zona da Mata (MG), determinada com simulador de chuva. Revista Brasileira de Ciência do Solo, v.5, p.7-14, 1981.

RODRIGUES DO Ó, N.C. Erodibilidade das principais classes de solo do Estado da Paraíba determinada por chuva simulada e método nomográfico. 1986. 29p. Dissertação (Graduação) Universidade Federal da Paraíba, Areia.

ROTH, C.H.; EGGERT, T. Mechanisms of aggregate breakdown involved in surface sealing, runoff generation and sediment concentration on loess soils. Soil \& Tillage Research, v.32, p.253-268, 1994.

SÁ, M.A.C. de. Energia ultra-sônica: uso e erodibilidade de solos. 2002. 95p. Tese (Doutorado) - Universidade Federal de Lavras, Lavras.

SÁ, M.A.C. de; LAGE, G.; LIMA, J.M. de. Calibração da potência emitida pelo sonificador para uso em análises de solo. Lavras: Ufla, 2001. 17p. (Boletim agropecuário, 41).

SÁ, M.A.C. de; LIMA, J.M. de; LAGE, G. Procedimento-padrão para medida da potência liberada pelo aparelho de ultra-som. Ciência e Agrotecnologia, v.24, p.300-306, 2000a.

SÁ, M.A.C. de; LIMA, J.M. de; MELLO, C.R. de. Nível de energia ultra-sônica para o estudo da estabilidade de agregados de um Latossolo sob diferentes usos. Pesquisa Agropecuária Brasileira, v.37, p.1649-1655, 2002.

SÁ, M.A.C. de; LIMA, J.M. de; SILVA, M.L.N.; DIAS JUNIOR, M. de S. Comparação entre métodos para o estudo da estabilidade de agregados em solos. Pesquisa Agropecuária Brasileira, v.35, p.1825-1834, 2000b.
SÁ, M.A.C. de; LIMA, J.M. de; SILVA, M.L.N.; DIAS JUNIOR, M.S. Índice de desagregação do solo baseado em energia ultra-sônica. Revista Brasileira de Ciência do Solo, v.23, p.525-531, 1999.

SILVA, I.F.; ANDRADE, A.P. Relatório de pesquisa sobre conservação do solo: 1977-1984. Areia: Sudene/UFPB, 1994. Não paginado.

SILVA, I.F.; ANDRADE, A.P.; CAMPOS FILHO, O.R. Erodibilidade de seis solos do semi-árido paraibano obtida com chuva simulada e método nomográfico. Revista Brasileira de Ciência do Solo, v.10, p.283-287, 1986.

SILVA, J.R.C. Erodibilidade dos solos do Ceará: distribuição espacial e avaliação de métodos para sua determinação $\left(1^{\mathrm{a}}\right.$ aproximação). 1994. 60p. Tese (Professor Titular) - Universidade Federal do Ceará, Fortaleza.

SILVA, M.L.N.; CURI, N.; FERREIRA, M.M.; LIMA, J.M. de; FERREIRA, D.F. Proposição de modelos para estimativa da erodibilidade de latossolos brasileiros. Pesquisa Agropecuária Brasileira, v.34, p.2287-2298, 1999.

TIPPKÖTTER, R. The effect of ultrasound on the stability of mesoaggregates $(60-200 \mu \mathrm{m})$. Zeitscchrift Pflanzenernährung und Bodenkunde, v.157, p.99-104, 1994.

UEHARA, G. Acric properties and their significance to soil classification. In: INTERNATIONAL SOIL CLASSIFICATION WORKSHOP, 8., 1986, Rio de Janeiro. Proceedings. Rio de Janeiro: Embrapa-SNLCS, 1988. Part 1, p.19-22.

ZELASNY, L.W.; HE, L.; VANWORMHOUDT, A. Charge analysis of soils and anion exchange. In: METHODS of soil analysis. Madison: SSSA, 1996. v.5, part 3, p.1231-1253.

Recebido em 18 de dezembro de 2003 e aprovado em 13 de abril de 2004 